\section{Mobility Degradation of 28-nm Bulk MOSFETs Irradiated to Ultrahigh Total lonizing Doses}

C.-M. Zhang*1 ${ }^{* 1}$ F. Jazaeri ${ }^{1}$, G. Borghello ${ }^{2,3}$, S. Mattiazzo ${ }^{4}$, A. Baschirotto ${ }^{5}$, and C. Enz ${ }^{1}$

${ }_{1}^{1}$ Integrated Circuits Laboratory, École Polytechnique

Fédérale de Lausanne, Neuchâtel 2002, Switzerland

2EP-ESE Group, CERN, Geneva 1211, Switzerland

${ }^{3}$ DPIA, University of Udine, Udine 33100 , Italy

${ }^{4}$ INFN-Padova \& University of Padova, Padova 35131, Italy

5 INFN-Milano \& University of Milano-Bicocca, Milano

20126, Italy

*E-mail: chunmin.zhang@epfl.ch

Abstract-Using the $\boldsymbol{Y}$-function method, this paper experimentally investigates the effects of total ionizing dose up to $1 \mathrm{Grad}$ on the channel mobility of a commercial $28-\mathrm{nm}$ bulk CMOS process.

Keywords-Interface traps, mobility degradation, oxide-trapped charges, shallow trench isolation, total ionizing dose, $28-\mathrm{nm}$ bulk MOSFETs, $Y$ function

\section{INTRODUCTION}

The total amount of energy absorbed by a MOSFET from incident ionizing radiation, measured by total ionizing dose (TID) using either a unit called rad or the SI unit called Gy $(1 \mathrm{~Gy}=100 \mathrm{rad}=1 \mathrm{~J} / \mathrm{kg}$ ), can build up an amount of trapped charges in oxides and activate hydrogen-passivated interface traps at silicon/oxide interfaces [1]. TID-induced oxide- and interface-trapped charges influence electrical characteristics of electronic devices [2]. For example, it gives rise to a drive current loss by increasing the absolute threshold voltage and/or reducing the effective channel mobility [3]. The innermost electronics of CERN's forthcoming high-luminosity Large Hadron Collider (HL-LHC) is anticipated to experience an unprecedented radiation level up to $1 \mathrm{Grad}$ of TID and $10^{16}$ neutrons $/ \mathrm{cm}^{2}$ of hadron fluence over ten years of operation [4]. This might lead to device degradation and even failure [5]. With the perspective of using advanced CMOS technologies in the HL-LHC, we have been studying the radiation tolerance of a commercial 28-nm bulk CMOS process up to $1 \mathrm{Grad}$ [3], [6]. Using the $Y$-function method [7], this paper experimentally investigates the effects of TID on the channel mobility in linear operation.

\section{Channel mobility eXtraction}

This work studies the drain-to-source current $I_{D S}$ in strong inversion of linear operation at a small value of drain-to-source voltage $\left(V_{D S}=0.01 \mathrm{~V}\right)$. Using the drift-diffusion model for drain current $I_{D S}=W \mu_{e f f} C_{o x}\left(V_{G B}-V_{T}\right) V_{D S} / L$ and the firstorder model for mobility degradation $\mu_{\text {eff }}=\mu_{0} /\left[1+\theta\left(V_{G B}-\right.\right.$ $\left.V_{T}\right)$ ] gives the current-voltage expression:

$$
I_{D S}=\frac{W}{L} \frac{\mu_{0}}{1+\theta\left(V_{G B}-V_{T}\right)} C_{o x}\left(V_{G B}-V_{T}\right) V_{D S},
$$

where $W$ and $L$ are the effective channel width and length, respectively, $\mu_{\text {eff }}$ is the effective channel mobility, $\mu_{0}$ is the lowfield channel mobility, $\theta$ is the effective mobility degradation coefficient, $V_{G B}$ is the gate-to-bulk voltage, $V_{T}$ is the charge threshold voltage, and $C_{o x}$ is the gate oxide capacitance per

This work is part of the GigaRadMOST project funded by the SNSF under grant number 200021_160185, in collaboration with the ScalTech28 project funded by the INFN. unit area. Differentiating $I_{D S}$ versus $V_{G B}$ for $G_{m}$ and combining it with (1), we obtain the well-known $Y$ function [7]:

$$
\frac{I_{D S}}{\sqrt{G_{m}}}=\sqrt{\frac{W}{L} \mu_{0} C_{o x} V_{D S}}\left(V_{G B}-V_{T}\right) .
$$

In strong inversion, $I_{D S} / \sqrt{G_{m}}$ is independent of $\theta$ and proportional to $V_{G B}$, as evidenced by the $\left|I_{D S}\right| / \sqrt{G_{m}}$ versus $\left|V_{G B}\right|$ curves in Fig. 1a-b and Fig. 1e-f. $V_{T}$ is defined as the $\left|V_{G B}\right|-$ intercept of the linear extrapolation and $\mu_{0}$ is deduced from the slope of the extrapolated line. At high TID levels, narrowchannel MOSFETs demonstrate a higher $V_{T}$ shift and a more significant $\mu_{0}$ reduction than wide-channel MOSFETs.

To get an accurate extraction of $V_{T}, \mu_{0}$, and $\theta$, this linear extrapolation has to be applied in a sufficient strong inversion region [7]. This can be checked by the occurrence of the $\theta\left(\left|V_{G B}\right|\right)$ plateau at high values of $\left|V_{G B}\right|$. Combining the derived $G_{m}$ with (1) enables us to extract $\theta$ :

$$
\theta=\left[\frac{I_{D S}}{G_{m}\left(V_{G B}-V_{T}\right)}-1\right] \frac{1}{V_{G B}-V_{T}}
$$

Fig. 1c-d and Fig. 1g-h confirm the $\left|V_{G B}\right|$ independence of $\theta$ in strong inversion. Due to the significant $V_{T}$ shift at high TID levels, narrow-channel $p$ MOSFETs have a reduced $\left|V_{G B}\right|$ range with the validity of the $Y$-function method. Furthermore, the corresponding $\theta$ tends to be zero, indicating a negligible influence of $\left|V_{G B}\right|$ on the $\mu_{e f f}$ of narrow-channel $p$ MOSFETs.

\section{GIGARAD-TID EFFECTS ON CHANNEL MOBILITY}

Fig. 2 plots the calculated $\mu_{\text {eff }}$ using the extracted $V_{T}, \mu_{0}$, and $\theta$ versus $\left|V_{G B}\right|-V_{T}$. We observe a $\left|V_{G B}\right|$-induced mobility degradation, except for narrow-channel $p$ MOSFETs that display a $\left|V_{G B}\right|$-independent $\mu_{e f f}$ at high TID levels. This corresponds to their negligible $\theta$ at high TID levels. We also observe a higher $\mu_{\text {eff }}$ reduction for narrow-channel MOSFETs than wide-channel MOSFETs. Note that $\mu_{0}$ corresponds to $\left|V_{G B}\right|-V_{T}=0$. As expected, the low-field electron mobility is around three times the low-field hole mobility.

The $\mu_{0}$ and $V_{T}$ variation is plotted versus TID in Fig. 3. We see a limited influence on $\mu_{0}(<20 \%)$ and $V_{T}(<7 \%)$ of wide-channel MOSFETs even at ultrahigh TID levels. This indicates the improved radiation tolerance of the advanced gate stacks. However, narrow-channel MOSFETs undergo a significant $\mu_{0}$ reduction up to $36 \%$ for $n$ MOSFETs (Fig. 3a) and up to $73 \%$ for $p$ MOSFETs (Fig. 3c). This is because narrowchannel MOSFETs are more sensitive to the TID-induced charge trapping related to the thick STI oxides. Near threshold, oxide-trapped charges near silicon/STI interfaces and trapped charges at silicon/STI interfaces act as Coulomb scattering centers that are believed to contribute to this significant mobility degradation. For narrow-channel $n$ MOSFETs, the negative $V_{T}$ shift (Fig. 3b) mitigates the effect of $\mu_{0}$ reduction on the drive current. However, for narrow-channel $p$ MOSFETs, oxideand interface-trapped charges together increase $V_{T}$ up to $36 \%$ (Fig. 3d) and degrade $\mu_{0}$ up to $73 \%$ that considerably reduces the drive current and leads to a much worse situation.

\section{CONClusion}

Using the $Y$-function method, we extract the channel mobility of commercial 28-nm bulk MOSFETs in strong inversion of linear operation before irradiation and at different TID levels. Extracted results confirm the enhanced radiation tolerance of wide-channel MOSFETs that is mostly due to the advanced 

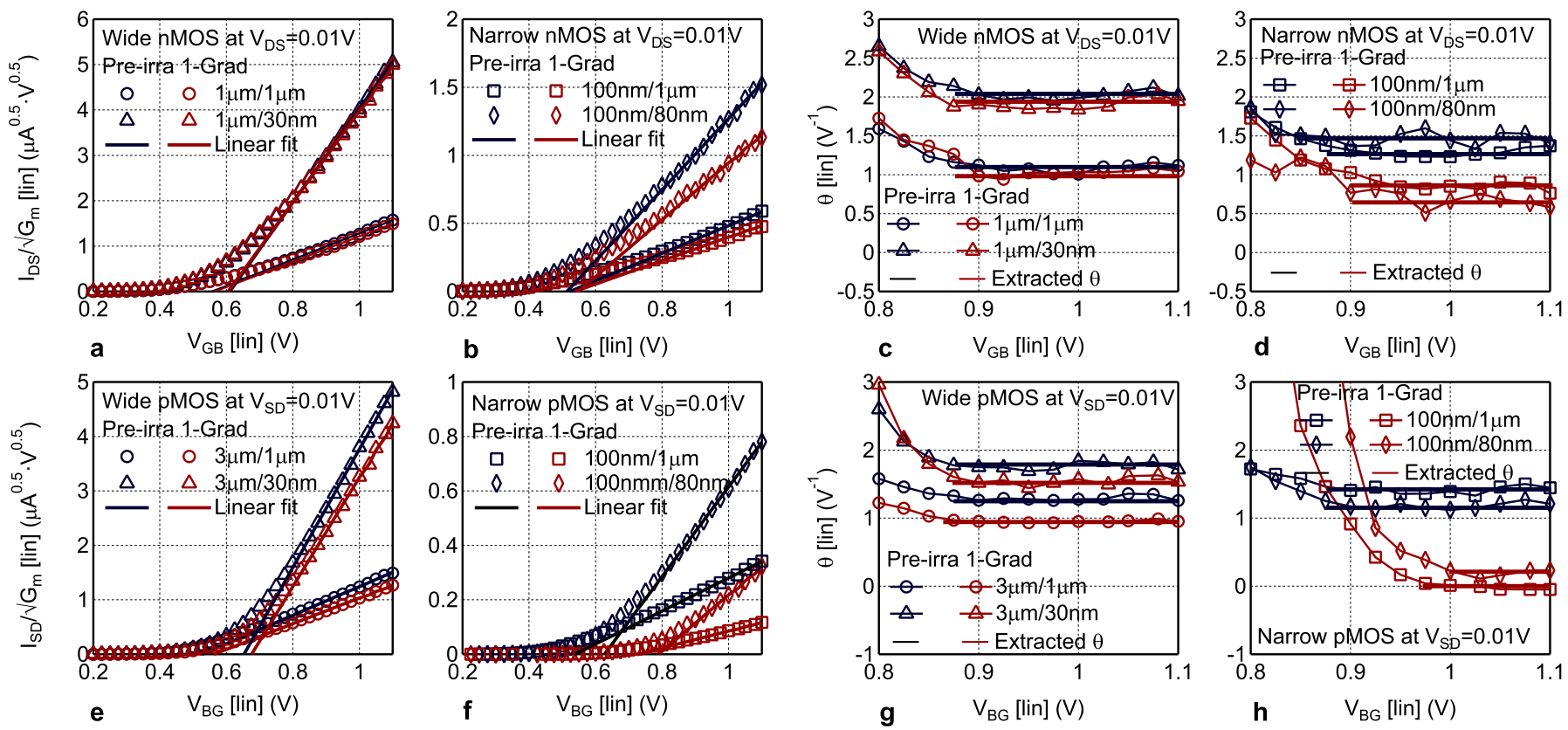

Fig. 1: $Y$-function $\left|I_{D S}\right| / \sqrt{G_{m}}$ (abef) and mobility degradation coefficient $\theta$ (cdgh) of $n$ - (a-d) and $p$ MOSFETs (e-h) versus gate-to-bulk voltage $\left|V_{G B}\right|$.
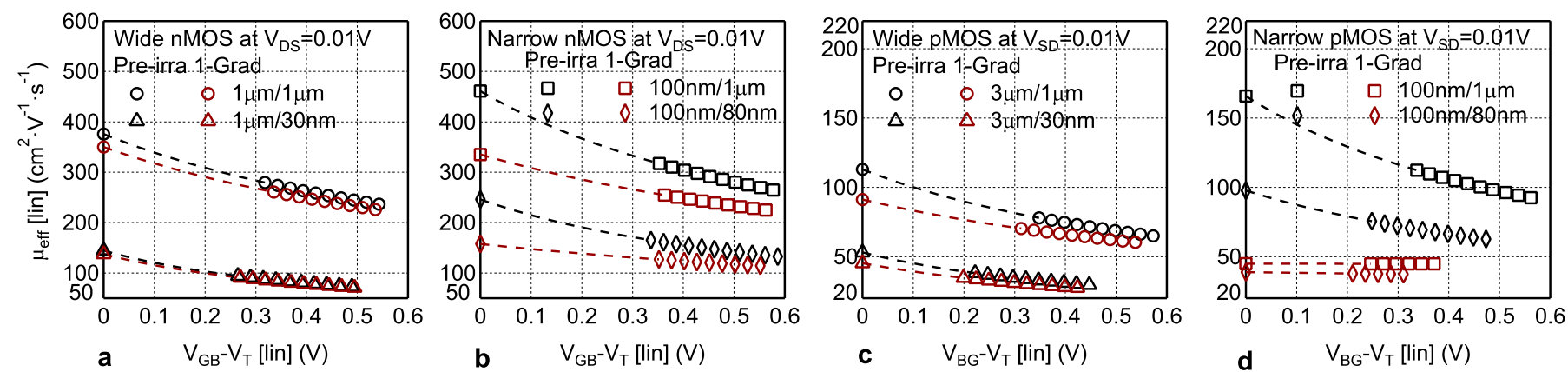

Fig. 2: Effective channel mobility $\mu_{\text {eff }}$ of $n$ - (ab) and $p$ MOSFETs (cd) versus overdrive voltage $\left|V_{G B}\right|-V_{T}$.

gate stacks in nanoscale CMOS technologies. Narrow-channel MOSFETs demonstrate a rather high mobility reduction that is attributed to the Coulomb scattering of the charge buildup related to the thick shallow trench isolation oxides.

\section{REFERENCES}

[1] H. J. Barnaby, "Total-ionizing-dose effects in modern CMOS technologies," IEEE Transactions on Nuclear Science, vol. 53, no. 6, pp. 3103-3121, Dec 2006.

[2] I. S. Esqueda, H. J. Barnaby, K. E. Holbert, F. El-Mamouni, and R. D. Schrimpf, "Modeling of ionizing radiation-induced degradation in multiple gate field effect transistors," IEEE Transactions on Nuclear Science, vol. 58, no. 2, pp. 499-505, April 2011.

[3] C.-M. Zhang, F. Jazaeri, A. Pezzotta, C. Bruschini, G. Borghello, F. Faccio, S. Mattiazzo, A. Baschirotto, and C. Enz, "Characterization of Gigarad total ionizing dose and annealing effects on 28-nm bulk MOSFETs," IEEE Transactions on Nuclear Science, vol. 64, no. 10, pp. 2639-2647, Oct 2017.

[4] T. A. Collaboration, "Technical Design Report for the ATLAS Inner Tracker Pixel Detector," CERN-LHCC-2017-021, ATLAS-TDR-030, Tech. Rep., 2017.

[5] D. M. Fleetwood, "Evolution of Total Ionizing Dose Effects in MOS Devices with Moore's Law Scaling," IEEE Transactions on Nuclear Science, vol. 65, no. 8, pp. 1465-1481, Aug 2018.

[6] C.-M. Zhang, F. Jazaeri, G. Borghello, F. Faccio, S. Mattiazzo, A. Baschirotto, and C. Enz, "Characterization and modeling of gigarad-tid-induced drain leakage current of 28-nm bulk MOSFETs," IEEE Transactions on Nuclear Science, 2018.

[7] G. Ghibaudo, "New method for the extraction of MOSFET parameters," Electronics Letters, vol. 24, no. 9, pp. 543-545, April 1988.
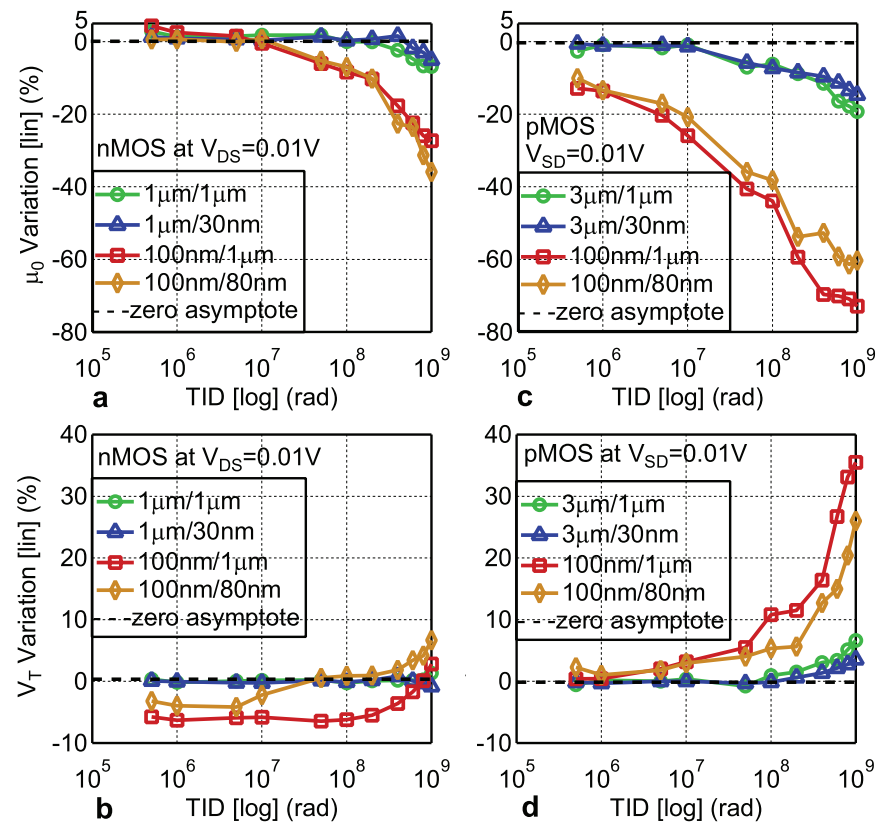

Fig. 3: Variation of low-field channel mobility $\mu_{0}$ (ac) and threshold voltage $V_{T}$ (bd) of $n$ - (ab) and $p$ MOSFETs (cd). 